\title{
QUEEN'S
UNIVERSITY
BELFAST
}

\section{Encapsulated doxorubicin crystals influence lysolipid temperature- sensitive liposomes release and therapeutic efficacy in vitro and in vivo}

Ruiz, A., Ma, G., Seitsonen, J., Pereira, S., Ruokolainen, J., \& Al-Jamal, W. T. (2020). Encapsulated doxorubicin crystals influence lysolipid temperature-sensitive liposomes release and therapeutic efficacy in vitro and in vivo. Journal of controlled release : official journal of the Controlled Release Society.

https://doi.org/10.1016/j.jconrel.2020.09.032

Published in:

Journal of controlled release : official journal of the Controlled Release Society

Document Version:

Peer reviewed version

Queen's University Belfast - Research Portal:

Link to publication record in Queen's University Belfast Research Portal

\section{Publisher rights}

Copyright 2020 Elsevier.

This manuscript is distributed under a Creative Commons Attribution-NonCommercial-NoDerivs License

(https://creativecommons.org/licenses/by-nc-nd/4.0/), which permits distribution and reproduction for non-commercial purposes, provided the author and source are cited.

\section{General rights}

Copyright for the publications made accessible via the Queen's University Belfast Research Portal is retained by the author(s) and / or other copyright owners and it is a condition of accessing these publications that users recognise and abide by the legal requirements associated with these rights.

Take down policy

The Research Portal is Queen's institutional repository that provides access to Queen's research output. Every effort has been made to ensure that content in the Research Portal does not infringe any person's rights, or applicable UK laws. If you discover content in the

Research Portal that you believe breaches copyright or violates any law, please contact openaccess@qub.ac.uk. 


\section{Encapsulated doxorubicin crystals influence lysolipid temperature-sensitive liposomes release and therapeutic efficacy in vitro and in vivo}

Amalia Ruiz ${ }^{1}$, Guanglong $\mathrm{Ma}^{1}$, Jani Seitsonen², Sara Pereira ${ }^{1 \dagger}$, Janne Ruokolainen², and Wafa' T. Al-Jamal ${ }^{1 *}$

1School of Pharmacy - Queen's University Belfast, Belfast, BT9 7BL, United Kingdom. 2Department of Applied Physics, Aalto University School of Science, P.O. Box 15100, FI-00076 Aalto, Finland.

Present address:

† AstraZeneca, Advanced Drug Delivery Group, Pharmaceutical Sciences, Aaron Klug Building, Granta Park, Cambridge CB21 6GH, UK.

* Author for correspondence:

Dr. Wafa' T. Al-Jamal

School of Pharmacy

Queen's University Belfast

Belfast, BT9 7BL

United Kingdom

E-mail:w.al-jamal@qub.ac.uk 


\begin{abstract}
Doxorubicin (DOX)-loaded lysolipid temperature-sensitive liposomes (LTSLs) are a promising stimuliresponsive drug delivery system that rapidly releases DOX in response to mild hyperthermia (HT). This study investigates the influence of loaded DOX crystals on the thermosensitivity of LTSLs and their therapeutic efficacy in vitro and in vivo. The properties of DOX crystals were manipulated using different remote loading methods (namely $\left(\mathrm{NH}_{4}\right)_{2} \mathrm{SO}_{4}, \mathrm{NH}_{4}$-EDTA and $\mathrm{MnSO}_{4}$ ) and varying the lipid:DOX weight ratio during the loading step. Our results demonstrated that $\left(\mathrm{NH}_{4}\right)_{2} \mathrm{SO}_{4}$ or $\mathrm{NH}_{4}$-EDTA remote loading methods had a comparable encapsulation efficiency (EE\%) into LTSLs in contrast to the low DOX EE\% obtained using the metal complexation method. Cryogenic transmission electron microscopy (cryoTEM) revealed key differences in the nature of DOX crystals formed inside LTSLs based on the loading buffer or/and the lipid:DOX ratio used, resulting in different DOX release profiles in response to mild $\mathrm{HT}$. The in vitro assessment of DOX release/uptake in CT26 and PC-3 cells revealed that the use of a high lipid:DOX ratio exhibited a fast and controlled release profile in combination with mild $\mathrm{HT}$, which correlated well with their cytotoxicity studies. Similarly, in vivo DOX release, tumour growth inhibition and mice survival rates were influenced by the physicochemical properties of LTSLs payload. This study demonstrates, for the first time, that the characteristics of DOX crystals loaded into LTSLs, and their conformational rearrangement during $\mathrm{HT}$, are important factors that impact the TSLs performance in vivo.
\end{abstract}

Keywords: lysolipid temperature-sensitive liposomes, hyperthermia, drug release, cancer therapy, doxorubicin, remote loading. 


\section{Introduction}

Delivering effective therapeutic doses to target sites whilst reducing systemic toxicity is a prerequisite to increase the selectivity of cancer treatments [1]. Nanomedicines have gained special attention in cancer therapy by altering anticancer drugs' pharmacokinetics and pharmacodynamics. Doxil ${ }^{\circledR}$ is a liposomal formulation of doxorubicin (DOX) that exhibits lower systemic adverse effects and reduced cardiotoxicity in cancer patients compared to free DOX [2]. Although the mechanisms of DOX-induced cardiotoxicity are not fully understood yet, it was shown in clinical studies that liposomal encapsulation of DOX have shown to provide a protective effect against cardiotoxicity by reducing myocardial drug accumulation [2], [3] and upregulating the expression of cardioprotective mechanisms [4]. Despite its extended blood circulation and increased tumour accumulation, Doxil ${ }^{\circledR}$ has shown limited therapeutic efficacy due to a low DOX bioavailability at the tumour site [5]. To circumvent this limitation, temperature triggerable liposomes have been developed enabling localised on-demand drug release and increasing the accumulation of anticancer drugs at the tumour site [6]. Temperature-sensitive liposomes (TSLs) were first reported by Yatvin et al. [7]. Afterwards, Needham et al., developed lysolipid-containing temperature-sensitive liposomes (LTSLS) with a burst release kinetic in response to mild hyperthermia $(\mathrm{HT})\left(41-42^{\circ} \mathrm{C}\right)[8]-[10]$. ThermoDox ${ }^{\circledR}$ is currently the most clinically advanced LTSL formulation, which consists of DPPC, MSPC (lysolipid) and DSPE-PEG 2000 (86:10:4 molar ratio) [11]. It has been used in combination with radio frequency ablation (RAF) to treat liver cancer (Phase III clinical trial) and recurrent regional breast cancer (Phase II clinical trial) [12]. More recently, ThermoDox ${ }^{\circledR}$ has been used in combination with high-intensity focused ultrasound (HIFU) to treat incurable hepatic tumours [13] and paediatric refractory solid tumours [14].

To date, stability and drug release from TSLs have been manipulated by varying the composition of the lipid bilayer to match the desired release profile [9], [10], [15]-[17]. Ultrafast DOX release from LTSLs is a prerequisite for intravascular release in heated tumours, while TSLs with slower release kinetics are more suitable for interstitial drug release [18], [19]. ThermoDox ${ }^{\circledR}$ has been prepared using a pHgradient remote loading method $\left[\left(\mathrm{NH}_{4}\right)_{2} \mathrm{SO}_{4}\right]$ at a 20:1 lipid:DOX weight ratio and displays a burst DOX release kinetic [9]. As the lipid bilayer composition is considered a key parameter to control drug release, the nature of the loaded drug should be equally important in influencing the overall release profile, and the therapeutic efficacy of TSLs formulations. In support of the latter assumption, the present work investigates the nature of the loaded DOX crystals on the thermosensitivity of LTSLs and their therapeutic efficacy in vitro and in vivo. The properties of DOX crystals were systematically manipulated using different remote loading methods (namely $\left(\mathrm{NH}_{4}\right)_{2} \mathrm{SO}_{4}, \mathrm{NH}_{4}-\mathrm{EDTA}$ and $\mathrm{MnSO}_{4}$ ) and varying the lipid:DOX weight ratio during the loading step. The in vitro assessment of DOX release/uptake in cancerous cells, in combination with mild HT, aimed to compare the influence of the loading buffer or/and the lipid:DOX ratio used, on the cytotoxicity of LTSLs. Similarly, our in vivo data explored the significant influence of DOX crystals on the intravascular release of DOX in combination with mild HT on tumour growth in vivo. The present study provides novel findings on how LTSLs DOX crystals' nature, dictated by the loading method and the lipid:DOX weight ratio, and their conformational 
rearrangement during $\mathrm{HT}$, impact the efficacy of DOX-loaded LTSLs in biological systems. Our work has highlighted, for the first time, the influence of loaded DOX on the overall performance of TSLs in vivo.

\section{Materials and Methods}

\section{Materials}

1,2-distearoyl-sn-glycero-3 phosphoethanolamine-N-[amino(polyethyleneglycol)-2000] (DSPEPEG ${ }_{2000}$ ) and 1,2-dipalmitoyl-sn-glycero-3-phosphocholine (DPPC), and were a kind gift from Lipoid GmbH (Ludwigshafen, Germany).1-stearoyl-2-hydroxy-sn-glycero-3-phosphocholine (MSPC) was purchased from Avanti Polar Lipid (Alabama, USA). Sodium chloride ( $\mathrm{NaCl}$ ), 4-(2-hydroxyethyl) piperazine-1-ethanesulfonic acid (HEPES), ethylenediaminetetraacetic acid (EDTA), ammonium sulphate $\left(\left(\mathrm{NH}_{4}\right)_{2} \mathrm{SO}_{4}\right)$, sucrose, manganese (II) sulphate $\left(\mathrm{MnSO}_{4}\right)$, and resazurin reagent (R7017) were obtained from Sigma-Aldrich (UK). Methanol (HPLC grade), chloroform (HPLC grade), and sterile 0.2 $\mu \mathrm{m}$ pore size syringe filters were purchased from Thermo Fisher Scientific (UK). Doxorubicin hydrochloride (DOX-HCL) was obtained from Apollo Scientific Ltd (Manchester, UK). Roswell Park Memorial Institute (RPMI) 1640 Medium, Dulbecco's PBS (1X), penicillin-streptomycin solution liquid (10000 units/mL), GlutaMAXTM supplement 200 mM, 0.05\% trypsin/EDTA, trypan blue stain (0.4\%) and were purchased from Invitrogen Gibco Life Technologies (UK). Foetal Bovine Serum (FBS), non-heat inactivated (10270106) or heat inactivated (10500064) was obtained from Gibco Life Technologies (UK).

\section{Methods}

\section{Liposome preparation}

Phospholipids were dissolved in a mixture of chloroform and methanol $(4: 1 \mathrm{v} / \mathrm{v})$ and placed in a $25 \mathrm{~mL}$ round-bottom flask. Liposomes were prepared by the lipid film hydration method. Briefly, the organic solvents were evaporated under reduced pressure at $60^{\circ} \mathrm{C}$ for 1 hour using a rotary evaporator $(\mathrm{BÜCHI}$, Labortechnik $A G$ ) and then flushed with $\mathrm{N}_{2}$ stream to remove any residual traces of organic solvent. To achieve a final lipid concentration of $5 \mathrm{mM}$, the dried lipid films of LTSL (DPPC:MSPC:DSPE-PEG 2000 86:10:4 molar ratio) were hydrated with one of the following buffers: ammonium sulphate pH 5.4 (240 $\mathrm{mM}\left(\mathrm{NH}_{4}\right)_{2} \mathrm{SO}_{4}$ ), EDTA diammonium salt pH 5.5 (300 mM NH${ }_{4} \mathrm{EDTA}$ ) or manganese sulphate buffer

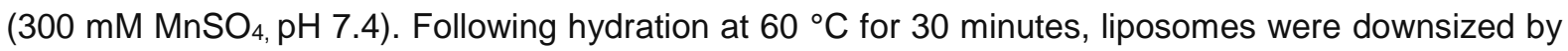
extrusion through $0.8 \mu \mathrm{m}$ (5 cycles), $0.2 \mu \mathrm{m}$ (15 cycles) and $0.1 \mu \mathrm{m}$ ( 20 cycles) polycarbonate filters using a mini-extruder (Avanti Polar Lipids). Liposomes were then flushed with $\mathrm{N}_{2}$ and allowed to anneal overnight at room temperature (RT). LTSLs using these different buffers will be abbreviated as L$\left(\mathrm{NH}_{4}\right)_{2} \mathrm{SO}_{4}$, L-NH $4-E D T A$, and L-MnSO 4 , respectively.

\section{Remote loading of DOX into LTSLS}

L- $\left(\mathrm{NH}_{4}\right)_{2} \mathrm{SO}_{4}$ or L-NH$H_{4}$-EDTA were exchanged with HEPES buffer saline (HBS, $20 \mathrm{mM}$ HEPES and 0.8 $w t \% \mathrm{NaCl}, \mathrm{pH}$ 8.5) using a Sephadex ${ }^{\mathrm{TM}}$ G-25 PD-10 column (GE Healthcare Life Sciences, UK). L- 
$\mathrm{MnSO}_{4}$ were exchanged with SHE buffer (300 mM sucrose, 20 mM HEPES and 15 mM EDTA, pH 7.4). After buffer exchange, LTSLs were incubated with DOX-HCl at various lipid:DOX weight ratios (20:1, 10:1, 5:1 and 2:1) at $37^{\circ} \mathrm{C}$ for 90 minutes. Following incubation, unencapsulated DOX was removed using a PD-10 column. DOX Encapsulation efficiency (EE) was determined by measuring fluorescence intensity at $\lambda_{\mathrm{ex}}=485 \mathrm{~nm}$ and $\lambda_{\mathrm{em}}=590 \mathrm{~nm}$ using a FLUOstar ${ }^{\mathrm{TM}}$ Omega Multimode Plate Reader (BMG Labtech, Germany). DOX-loaded LTSLs before and after purification were lysed with $1 \mathrm{wt} \%$ Triton X100 (0.1 wt.\% after dilution), and DOX EE\% was calculated by Equation 1:

$$
D O X E E \%=\left(l_{(t)} \text { after purification }\right) /\left(l_{(t)} \text { before purification }\right)^{*} 100 \quad \text { Equation } 1
$$

\section{Dynamic Light Scattering (DLS)}

Hydrodynamic diameter and $\zeta$-potential (ZP) measurements were performed using a Zetasizer NanoZS90 (Malvern, UK). Size and ZP measurements were performed using disposable polystyrene cells and disposable plain folded capillary zeta cells (Malvern, UK), respectively. LTSLs samples were diluted by either 100 or 20 -fold in deionized water; the former for size measurements and the latter for ZP measurements. All measurements were performed at $25 \stackrel{\circ}{\circ}$. Size measurements were performed with 3 measurements each with 15 scans, while ZP measurements were performed with 6 measurements each with 20 scans.

\section{Cryo-Transmission Electron Microscopy (Cryo-TEM)}

Morphological characterisation of empty and DOX-loaded LTSLs was carried out using Cryo-TEM. To assess the effect of hyperthermia on DOX crystals, the samples were placed in a $42{ }^{\circ} \mathrm{C}$ water bath for 1 hour. A drop of $5 \mu \mathrm{L}$ of the sample was deposited on Quantifoil R 2/1 200 mesh holey carbon-coated copper grids. The excess solution was removed by blotting for 3 seconds in $80 \%$ relative humidity using an automatic plunge freezer (EM GP2, Leica Microsystem), followed by immediate vitrification using liquid ethane $\left(-175^{\circ} \mathrm{C}\right)$. Vitrified samples were cryo-transferred to the microscope and imaged using a JEOL JEM-3200 FSC TEM while maintaining specimen temperature at $-187^{\circ} \mathrm{C}$. The size and shape distributions were determined by manual measurement of more than 100 particles using the public domain software ImageJ (NIH, Bethesda, MD, USA, https://imagej.nih.gov/ij/).

\section{DOX release study}

The release of DOX from LTSLs was assessed at both $37^{\circ} \mathrm{C}$ and $42^{\circ} \mathrm{C}$ in $\mathrm{HBS} \mathrm{pH} 7.4$ and $50 \%$ nonheat inactivated foetal bovine serum (FBS). Release studies were performed at various time points. For DOX release experiments, $50 \mu \mathrm{L}$ samples were taken at each time point and further diluted in $\mathrm{HBS} \mathrm{pH}$ 7.4 to a final volume of $200 \mu \mathrm{L}$. Samples were then transferred into a 96-well black, clear flat-bottom plate, and fluorescence intensity was quantified at $\lambda_{\mathrm{ex}}=485 \mathrm{~nm}$ and $\lambda_{\mathrm{em}}=590 \mathrm{~nm}$ using a FLUOstar ${ }^{\mathrm{TM}}$ Omega Multimode Plate Reader (BMG Labtech, Germany). The percentage of DOX released at each time point was calculated by Equation 2:

$$
\text { DOX release } \left.\left.(\%)=\left(\left[I_{(s)}\right)^{-} I_{(0)}\right]\right) /\left(\left[I_{(t)}\right)^{-I}(0)\right]\right) \times 100 \quad \text { Equation } 2
$$


Where $I_{(s)}$ is the fluorescence intensity of samples at various time points, $I_{(0)}$ is the fluorescence intensity of Dox-loaded liposomes (background), and $I_{(t)}$ is the fluorescence intensity of the liposome suspension at time $\mathrm{t}=0$ hours after lysis in $0.1 \%(\mathrm{w} / \mathrm{v})$ Triton $^{\mathrm{TM}} \mathrm{X}-100$.

\section{Cell culture}

Murine colon carcinoma cells (CT26) (CRL-2638) and human prostate adenocarcinoma derived from the bone metastatic site (PC-3) (CRL-1435) cells were obtained from American Type Culture Collection (ATCC, USA). Cells were cultured in RPMI 1640 (Invitrogen Gibco Life Technologies), supplemented with $10 \%$ heat inactivated FBS, $50 \mathrm{U} / \mathrm{mL}$ penicillin, $50 \mu \mathrm{g} / \mathrm{mL}$ streptomycin, and $1 \%$ L-glutamine and maintained in a humidified chamber at $37{ }^{\circ} \mathrm{C}$ and $5 \% \mathrm{CO}_{2}$.

\section{Cell uptake studies}

Cells were seeded in 12 -well plates $\left(1 \times 10^{5}\right.$ cells/ well, $1 \mathrm{~mL} /$ well). The next day, $2 \mu \mathrm{M}$ of free DOX or DOX-loaded LTSLs were prepared in $400 \mu \mathrm{L}$ of media, added to the cells and incubated for 1 hour at $37 \stackrel{\circ}{-} \mathrm{C}$ or $42 \stackrel{\circ}{\circ}$ (water bath). The incubation of the liposomes was assessed in media containing 10 or $50 \%$ FBS. 1-hour post incubation, the supernatant was aspirated and discarded, and the cells were washed twice with PBS and detached from the plates using a solution of $0.05 \%$ Trypsin/EDTA. Finally, the cells were pelleted in a microcentrifuge ( $300 \mathrm{~g}, 5$ minutes) and resuspended in $300 \mu \mathrm{L}$ of PBS, and immediately acquired on the cytometer (BD FACSCalibur ${ }^{\mathrm{TM}}$, Becton, Dickinson and Company, UK) using the FL1 channel, $\lambda_{\mathrm{em}}=530 \mathrm{~nm}$. At least 10,000 cells were counted for each sample and experiments were performed in triplicates. Flow cytometry data was generated using BD CellQuest Pro software. Values represent mean \pm SD.

For confocal microscopy, the cells were seeded in 8-well chamber slides (Thermo Scientific) $\left(1 \times 10^{4}\right.$ cells/ well, $500 \mu \mathrm{L} /$ well). The next day, $2 \mu \mathrm{M}$ of free DOX or DOX-loaded LTSLs were prepared in 200 $\mu \mathrm{L}$ of media and added to the cells. Cells were co-treated with mild HT (42 $\left.{ }^{\circ} \mathrm{C}\right)$ for 1 hour using a water bath and returned to $37^{\circ} \mathrm{C}$ for another hour. Then the drug was removed by washing the cells three times with PBS. Cells were fixed with $0.5 \mathrm{~mL}$ of $4 \%(\mathrm{v} / \mathrm{v})$ paraformaldehyde in PBS (RT for 15 minutes in the dark), then washed twice with PBS and counterstained with $1 \mu \mathrm{g} / \mathrm{mL}$ of Hoechst 33342 (ThermoFisher) for 10 minutes. Finally, the coverslips were mounted on glass-chamber slides using ProLong Gold Antifade mounting media (Invitrogen Life Technologies). Confocal images were acquired with a Leica SP8 microscope (Leica microsystems) using a 63X oil immersion objective and 405 and $488 \mathrm{~nm}$ laser detectors in the hybrid (HyD) mode. Image analysis was performed using the public domain FIJI software (https://imagej.net/Fiji).

\section{In vitro toxicity}

CT26 cells were trypsinised, stained with trypan blue $(0.4 \%, 1: 1 \mathrm{v} / \mathrm{v}$ ratio) and counted using a haemocytometer. Cells were seeded $\left(1 \times 10^{4}\right.$ cells/ well, $200 \mu \mathrm{L} /$ well) in 96-well culture plates in complete RPMI 1640 media. The next day, $2 \mu \mathrm{M}$ of free DOX or DOX-loaded LTSLs were prepared in 
$30 \mu \mathrm{L}$ of media and added to each well. Cells were incubated at $37^{\circ} \mathrm{C}$ or $42 \stackrel{\circ}{\circ} \mathrm{C}$ for 1 hour and then returned to $37^{\circ} \mathrm{C} .4$ hours post incubation, cells were washed 3 times with PBS and cell viability was assessed after 48 or 72 hours using resazurin assay. Cells were incubated with $0.01 \mathrm{mg} / \mathrm{mL}$ resazurin solution for 4 hours. After incubation, the media were collected and transferred to a black 96 -well plate and the fluorescence $\left(\lambda_{\mathrm{ex}}=544 \mathrm{~nm}, \lambda_{\mathrm{em}}=590 \mathrm{~nm}\right.$ ) was read using an automated FLUOstar Omega (BMG Labtech, UK) plate reader. Six replicates per condition were used. The results were expressed as the percentage of cell viability (mean $\pm \mathrm{SD}$ ) and normalised to control untreated cells.

\section{Tumour model}

Balb/c female mice, 5-6 weeks old, (15-20 g) were purchased from Envigo, UK. Animal procedures were performed in compliance with the UK Home Office Code of Practice for the Housing and Care of Animals used in Scientific Procedures. Mice were housed in groups of 5 with free access to food and water and kept at a temperature of $19-22{ }^{\circ} \mathrm{C}$ and relative humidity of $45-65 \%$. Before performing the procedures, animals were acclimatised to the environment for at least 7 days. CT26 solid tumours were established by subcutaneous injection of $2.5 \times 10^{5}$ murine colon carcinoma cells in a volume of $20 \mu \mathrm{L}$ of PBS into the lower legs (single or bifocal for therapy or release study, respectively) using $26 \mathrm{G}$ needles. The tumour volume was estimated by measuring the orthogonal diameters ( $a$ (length) and $b$ (width)) with a digital calliper. The volume was calculated as $a \times(b)^{2} \times 0.5 \mathrm{~mm}^{3}$.

\section{In vivo release study}

In order to study LTSLs' biodistribution and in vivo DOX release, mice were placed on Teklad Global 2019X for 5-7 days prior to imaging to reduce body autofluorescence. When the tumours reached a suitable size $\left(\sim 600 \mathrm{~mm}^{3}\right)$, mice were shaved (whole body) for imaging. Tumour-bearing mice were randomised and divided into groups ( $n=3$ per group). Mice were anesthetised by inhalational isoflurane and injected via the tail vein with $200 \mu \mathrm{L}$ of PBS (control) or DOX-loaded LTSLs (8 mg/kg of DOX, 2.5 $\mu \mathrm{mol}$ lipids) in HBS. Following the injection, one tumour-bearing leg of the anaesthetised mouse was immersed in a $42{ }^{\circ} \mathrm{C}$ water bath for 1 hour to trigger DOX release. The other tumour-bearing leg was used as a control. The body temperature was monitored using a heating pad and a rectal thermocouple. The biodistribution of the DOX-loaded LTSLs was assessed in vivo using an In-Vivo Xtreme imaging system (Bruker). Images were taken immediately after HT and 24 hours post-injection. At the end of the study, mice were culled and organs and tumours were excised and imaged. The excitation wavelength was set at $480 \mathrm{~nm}$ and the emission wavelength was set at $600 \mathrm{~nm}$ with an exposure time of 10 seconds. Images were analysed with Molecular Imaging software. For quantification, Region of Interest $(\mathrm{ROI})$ analysis was applied.

\section{In vivo therapy study}

When the tumours reached a suitable size $\left(\sim 100 \mathrm{~mm}^{3}\right)$, tumour-bearing mice were randomised and divided into groups ( $\mathrm{n}=6$ per group). Mice were anesthetised by inhalational isoflurane and injected via the tail vein with $200 \mu \mathrm{L}$ of PBS (control) or DOX-loaded LTSLs ( $5 \mathrm{mg} / \mathrm{kg}$ of DOX, $2.5 \mu \mathrm{mol}$ lipids) in HBS. Following the injection, the tumour-bearing leg of the anaesthetised mice were immersed in a 42 
${ }^{\circ} \mathrm{C}$ water bath for 1 hour. The body temperature was monitored using a heating pad and a rectal thermocouple. Body weight and tumour growth were measured three times per week, as described above.

\section{Statistical analysis}

GraphPad Prism 5.0 (Prism 5.0; GraphPad Software Inc, San Diego, CA) was used to perform the twoway analysis of variance (ANOVA) test when 3 or more groups were analysed. When statistically significant differences were found ( $P$ value $<0.05$ ), the analysis was complemented with the Bonferroni post-test method.

\section{Results}

\section{DOX-loaded LTSLs preparation using different remote loading methods}

LTSLS (DPPC:MSPC:DSPE-PEG $2000,86: 10: 4$ molar ratio) were prepared using the thin lipid film hydration and extrusion method. DOX was remotely loaded into the LTSLs using different $\mathrm{pH}$-gradient methods; $\left(\mathrm{NH}_{4}\right)_{2} \mathrm{SO}_{4}, \mathrm{NH}_{4}$-EDTA, or via metal complexation with $\mathrm{MnSO}_{4}$. DOX-loaded LTSLs using these different buffers are abbreviated as $\mathrm{L}-\left(\mathrm{NH}_{4}\right)_{2} \mathrm{SO}_{4}, \mathrm{~L}-\mathrm{NH}_{4}-\mathrm{EDTA}$, and $\mathrm{L}-\mathrm{MnSO}_{4}$, respectively. Various lipid:DOX weight ratios were investigated, and the colloidal properties of DOX-loaded LTSLS were determined by measuring their hydrodynamic size, PDI and $\zeta$-potential (ZP). The physicochemical properties of DOX-loaded LTSLs using the three remote-loading methods are summarised in Table 1. All prepared DOX-loaded LTSLs had comparable hydrodynamic diameters ranging between 140-160 $\mathrm{nm}$. A similar negative surface charge was recorded for all formulations $(-10 \mathrm{mV}$ to $-14 \mathrm{mV})$. The encapsulation efficiency (EE\%) was determined at 20:1, 10:1 . 5:1 and 2:1 lipid:DOX weight ratios for L$\left(\mathrm{NH}_{4}\right)_{2} \mathrm{SO}_{4}, \mathrm{~L}-\mathrm{NH}_{4}-\mathrm{EDTA}$ and L-MnSO 4 (Figure 1). L-( $\left(\mathrm{NH}_{4}\right)_{2} \mathrm{SO}_{4}$ exhibited comparable EE values to L$\mathrm{NH}_{4}$-EDTA. In those cases, DOX-loading showed consistently higher EE\% ( 90\%) across the various lipid:DOX ratios with an approximate $15 \%$ decrease from 20:1 to $2: 1$ ratio in the case of L-NH -EDTA $_{4}$ (Figure $1 \mathrm{a}, \mathrm{b}$ ). L-MnSO 4 (Figure $1 \mathrm{c}$ ) had the lowest EE\% ( 25\%) across all ratios. Visually, ${\mathrm{L}-\mathrm{NH}_{4}-}^{-}$ EDTA suspension (Figure $1 \mathrm{~d}$ ) and $\mathrm{L}-\left(\mathrm{NH}_{4}\right)_{2} \mathrm{SO}_{4}$ (not shown), exhibited an orange/red colour in contrast to the purple colour showed by $\mathrm{L}^{-\mathrm{MnSO}_{4}}$ liposomes (Figure $1 \mathrm{e}$ ), where the latter was attributed to the DOX-Mn ${ }^{2+}$ complex formation, as previously reported [20], [21].

Table 1\& Figure 1

\section{DOX crystals loaded into LTSLs exhibit different morphology based on the loading buffer and DOX content}

For this study, the DOX-loaded LTSLs' morphology and structure were analysed using cryotransmission electron microscopy (cryo-TEM). Cryo-TEM examination revealed significant differences in the morphology of DOX-loaded LTSLs, as well as the physical state of DOX crystals inside LTSLs, 
depending on the loading buffer and the lipid:DOX weight ratio used (Figure 2). The DOX crystals' arrangement (U-ring, bundle, thick ring or empty look) and DOX-loaded LTSL morphology (round or elongated shape) were quantitively determined, as shown in Figure 3.

Figure 2 \& Figure 3

DOX crystals in L- $\left(\mathrm{NH}_{4}\right)_{2} \mathrm{SO}_{4}(20: 1)$ and L-NH 4 -EDTA (20:1) appeared as rod-shaped or thin-ring bundles. Some empty-looking vesicles were also present (Figure 2, Figure $3 \mathrm{a}, \mathrm{b}$ ). This is the first report to visualise DOX-EDTA crystals in liposomes which looks similar to previously loaded Idarubicin-EDTA crystals in conventional liposomes [22]. Upon decreasing the lipid:DOX ratio, fewer empty vesicles were observed and the morphology of $\mathrm{L}-\left(\mathrm{NH}_{4}\right)_{2} \mathrm{SO}_{4}(2: 1)$ showed two different populations with a spherical or elongated appearance (Figure 2, Figure $3 \mathrm{~d}, \mathrm{e}$ ). Furthermore, increasing DOX content caused a significant thickening of DOX rings and bundles within the core of $\mathrm{L}-\left(\mathrm{NH}_{4}\right)_{2} \mathrm{SO}_{4}(2: 1)$. In the case of $\mathrm{L}$ $\mathrm{NH}_{4}$-EDTA (2:1), liposomes exhibited only a spherical appearance with thick DOX crystal rings (Figure 2, Figure $3 \mathrm{f}$ ). On the other hand, $\mathrm{L}^{-\mathrm{MnSO}_{4}}$ (20:1 and 2:1) presented a faceted shape and all the liposomes were empty-looking (Figure 2, Figure $3 \mathrm{c}, \mathrm{g}$ ), probably due to the lower DOX EE\% of this formulation (Figure $1 \mathrm{c}$ ). In conclusion, cryo-TEM images confirmed different liposomes' morphology and intraliposomal DOX crystal structure depending on the buffer and the lipid:DOX ratio used for DOX loading.

To assess the effect of HT on the liposomes' DOX release profile, samples were heated for 1 hour at $42^{\circ} \mathrm{C}$ and imaged with cryo-TEM. Our results showed that all DOX-loaded LTSLs loaded at 20:1 weight ratio revealed an empty-looking appearance under cryo-TEM (data not shown), indicating the fast dissolution of these thin DOX crystals [23]. In contrast, the heated L- $\left(\mathrm{NH}_{4}\right)_{2} \mathrm{SO}_{4}(2: 1)$ sample showed empty-looking vesicles but a few liposomes still showed some thin bundles, suggesting a partial dissolution of DOX crystal and incomplete DOX release (Figure 2, Figure $3 \mathrm{~h}$ ). Furthermore, heated L$\mathrm{NH}_{4}$-EDTA (2:1) showed vesicles containing DOX crystals precipitated as disordered needles (Figure 2 , Figure $3 \mathrm{i}$ ), which could be attributed to a lower solubility of these crystals in contrast to the DOXsulphate ones. The slow release of DOX from conventional liposomes loaded using $\mathrm{NH}_{4}$-EDTA buffer compared to a $\left(\mathrm{NH}_{4}\right)_{2} \mathrm{SO}_{4}$ buffer, was previously reported [24]. These structural differences in DOX crystals, and their rearrangement in response to $\mathrm{HT}$, were further investigated in vitro and in vivo in order to explain their impact on DOX release/uptake and toxicity.

\section{L-MnSO 4 exhibit lower thermosensitivity and stability than L-NH4-EDTA \& $\mathrm{L}-\left(\mathrm{NH}_{4}\right)_{2} \mathrm{SO}_{4}$}

To assess the thermosensitivity of DOX-loaded LTSLs using different remote loading methods, comparative release studies were performed with DOX-loaded LTSLS prepared at 20:1 and 2:1 lipid:DOX weight ratios. DOX release profiles from LTSLs were determined in $\mathrm{HBS} \mathrm{pH} 7.4$ or $50 \%$ serum at $42{ }^{\circ} \mathrm{C}$ and $37^{\circ} \mathrm{C}$ (Figure 4). Surprisingly, despite the obvious differences in DOX crystals observed 
by cryo-TEM, L-( $\left(\mathrm{NH}_{4}\right)_{2} \mathrm{SO}_{4}$ at weight ratios of both $20: 1$ and 2:1 exhibited burst DOX release $(95 \%$ release after 5 minutes) at $42{ }^{\circ} \mathrm{C}$ in HBS (Figure 4 a) and 50\% FBS (Figure 4 b). L-( $\left(\mathrm{NH}_{4}\right)_{2} \mathrm{SO}_{4}(20: 1)$ release results are in line with previously reported literature, due to the presence of the lysolipid in the formulation [9], [10]. Both L-( $\left(\mathrm{NH}_{4}\right)_{2} \mathrm{SO}_{4}$ and L-NH $4-E D T A$ formulations presented similar release profiles (Figure 4 c, d). In contrast, $\mathrm{L}-M n S O_{4}(20: 1)$ exhibited a slower release profile at $42{ }^{\circ} \mathrm{C}$, where $20-40 \%$ of DOX was released in the first 10 minutes, followed by a slow-release kinetic, reaching $70 \%$ after 30 minutes (Figure $4 \mathrm{e}, \mathrm{f})$. L-MnSO $4(2: 1)$ exhibited a significantly lower release than L-MnSO $4(20: 1)$ at 42 $\stackrel{\circ}{\circ}$.

Figure 4

The stability of all formulations at $37^{\circ} \mathrm{C}$ was also assessed. Overall, DOX-loaded LTSLs prepared at a 20:1 weight ratio exhibited partial DOX leakage at $37{ }^{\circ} \mathrm{C}$ in $50 \%$ serum (Figure 4, right panel), which is attributed to the destabilising effect of serum on lysolipid containing liposomes [25]. Surprisingly, the leakage was reduced in all DOX-loaded LTSLs at 2:1 weight ratio. This finding suggests that DOX release from LTSLs could be altered based on DOX loading buffers, lipid:DOX ratio, and the incubating media, resulting in different release profiles depending on the biological scenario; in vitro (10\% serum) or in vivo (50\% serum). $\mathrm{L}-\left(\mathrm{NH}_{4}\right)_{2} \mathrm{SO}_{4}(2: 1)$ and $\mathrm{L}-\mathrm{NH}_{4}$-EDTA (2:1) were stable at $37{ }^{\circ} \mathrm{C}$ in HBS with negligible DOX release $(<5 \%$ ) over 60 minutes (Figure 4 a, Figure $4 \mathrm{c}$ ). In contrast, L-MnSO 4 showed higher DOX leakage at $37^{\circ} \mathrm{C}$ in HBS (Figure $4 \mathrm{e}$ ), indicating the lower stability of this formulation even in the absence of serum proteins, which could be due to the presence of an amorphous non-crystalline DOX [26].

\section{LTSLs loaded at a low lipid:DOX ratio showed lower DOX in vitro release in combination with HT}

To assess the in vitro release of DOX-loaded LTSLs prepared with different remote loading methods, comparative release/uptake studies were performed with DOX-loaded LTSLs prepared at 20:1 and 2:1 lipid:DOX weight ratios. DOX release/uptake profiles in cell culture media, containing $10 \%$ or $50 \%$ serum, were evaluated in vitro in the absence $\left(37^{\circ} \mathrm{C}\right)$ and presence of 1 -hour $\mathrm{HT}\left(42^{\circ} \mathrm{C}\right)$ using murine colon carcinoma cells (CT26) (Figure 5) and human prostate adenocarcinoma (PC-3) (Figure SI 1). Both cell lines were incubated for 1 hour at $37^{\circ} \mathrm{C}$ or $42^{\circ} \mathrm{C}$ with $2 \mu \mathrm{M}$ of free DOX or DOX-loaded LTSLS prepared at 20:1 and 2:1 lipid:DOX weight ratios. Then, the cells were washed with PBS, trypsinised, and immediately acquired on the flow cytometer in order to determine the DOX uptake in these cell lines. It is worth mentioning that DOX uptake at $37^{\circ} \mathrm{C}$ was used to assess the stability of the formulations, since minimal DOX release, and thus cell uptake, are expected in cells incubated with stable formulations.

Figure 5 
Promisingly, our results showed that all of the formulations, except L-MnSO $4(20: 1)$, remained stable at $37^{\circ} \mathrm{C}$ in the presence of $10 \%$ or $50 \%$ serum. On the other hand, $\mathrm{L}^{-\mathrm{MnSO}_{4}}(20: 1)$ exhibited a significant DOX leakage and cellular uptake. Nevertheless, this result was anticipated since some instability of this formulation was observed in HBS buffer (Figure $4 \mathrm{e}, \mathrm{f}$ ). In contrast to DOX release in a cell-free setup and the different serum used (Figure 4), the thermosensitivity of our DOX-loaded LTSLs in vitro seems to be influenced primarily by the lipid:DOX weight ratio used, serum concentration in the media, and to a lesser extent the remote loading method. In support of this, significantly higher release/uptake of DOX was detected in the cells incubated with all DOX-loaded LTSLs prepared at 20:1 lipid:DOX weight ratio compared to 2:1 ratio, regardless of the method of loading. Also, by incubating the cells with the samples in cell culture media containing $50 \%$ of serum, the release/uptake of DOX from all LTSLs decreased, which could be attributed to the formation of a protein corona around the liposomes, making the lipid bilayer less permeable, as reported by others [27]. Previous studies have highlighted the importance of the protein corona composition and the surrounding environment on TSL thermosensisitivty and their drug release profile [28].

Finally, regarding the effect of the remote loading method used on DOX release in vitro, there was a noticeable difference in DOX release/uptake in cells incubated with the formulations prepared at 2:1 lipid:DOX weight ratio, with minimal differences between formulations prepared at 20:1 ratio. As evidenced in figure $4 \mathrm{~b}$, cells incubated with L-NH 4 -EDTA (2:1) showed no DOX uptake compared with $\mathrm{L}-\left(\mathrm{NH}_{4}\right)_{2} \mathrm{SO}_{4}(2: 1)$ and L-MnSO 4 (2:1). These results are in agreement with the cryo-TEM results, where HT triggered complete DOX release at 20:1 ratio from all formulations but not at the 2:1 ratio. Heated $\mathrm{L}-\left(\mathrm{NH}_{4}\right)_{2} \mathrm{SO}_{4}(2: 1)$ showed considerable DOX release, while the heated L-NH 4 -EDTA (2:1) exhibited a significant amount of DOX precipitated crystals inside the liposomes, indicating inefficient DOX release.

After assessing the in vitro release and cellular uptake using flow cytometry, we studied the intracellular fate of free DOX or DOX-loaded LTSLs in CT26 and PC-3 cells at an equivalent dose of $2 \mu \mathrm{M} \mathrm{DOX.}$ Since the fluorescence of DOX is not quenched after its release, this enabled tracking its subcellular localisation in CT26 and PC-3 cells using CLSM (Figure 6). The CLSM results confirmed the highest uptake by СT26 cells treated with the formulations prepared at 20:1 ratio compared to 2:1. Also, the micrographs depicted a significant difference in the DOX uptake for L-NH $4-E D T A ~(2: 1)$ and L-MnSO 4 (2:1) compared with $\mathrm{L}-\left(\mathrm{NH}_{4}\right)_{2} \mathrm{SO}_{4}(2: 1)$, where the latter showed the fastest release and uptake. $\mathrm{A}$ similar trend was observed in the release/uptake in PC-3 cells (Figure 6, bottom panels). The nuclear localisation of DOX in PC3 cells was faster than in CT26 cells, as previously reported [29], [30]; however, DOX-loaded LTSLs using different remote loading methods and lipid:DOX ratios exhibited a similar pattern of release/uptake in both cell lines.

\section{Figure 6}

Overall, faster DOX release was obtained in vitro using LTSLs loaded at 20:1 compared to 2:1. This release kinetic profile was determined by the DOX crystals' arrangement and their dissolution, as shown by cryo-TEM, which dramatically affected DOX uptake by the cells. These in vitro findings highlight the 
importance of the DOX loaded crystals' characteristics, as well as the crucial role of the surrounding media (e.g. serum content \& cells) in modulating LTSLs release/uptake profile.

\section{Remote loading methods and lipid:DOX ratio influence DOX-loaded LTSLs in vitro toxicity}

To determine if the cytotoxicity of DOX-LTSLs is influenced by the remote loading method used, the toxicity of $\mathrm{L}-\left(\mathrm{NH}_{4}\right)_{2} \mathrm{SO}_{4}$, L-NH $4-\mathrm{EDTA}$, or $\mathrm{L}-\mathrm{MnSO}_{4}$ was assessed in vitro in the absence $\left(37^{\circ} \mathrm{C}\right)$ and presence of 1 -hour $\mathrm{HT}\left(42^{\circ} \mathrm{C}\right)$. Murine colon carcinoma cells (CT26) were treated for 1 hour with $\mathrm{HT}$, with $2 \mu \mathrm{M}$ of free DOX or DOX-loaded LTSLs prepared at 20:1 and 2:1 lipid:DOX weight ratios. Three hours later, the cells were washed, and toxicity was assessed after 48 and 72-hours post-treatment using resazurin assay (Figure 7).

Figure 7

No toxicity was observed in all treated groups at $37^{\circ} \mathrm{C} 48$ hours post-treatment; however, higher toxicity was observed after 72 hours. The toxicity of free DOX in CT26 cells was significantly increased in combination with HT, which was superior to all DOX-loaded LTSLs formulations and was shown to be time-dependent. As expected, the toxicity of DOX-loaded LTSLs in CT26 cells was dependent on the loading method, the lipid:DOX weight ratio used for the liposome's preparation, and the incubation time. No differences were observed in the CT26 cells incubated with L- $\left(\mathrm{NH}_{4}\right)_{2} \mathrm{SO}_{4}(20: 1)$ or (2:1) (Figure 7 a, d), which agrees with their faster in vitro release/uptake profile compared with the other formulations (Figure $5 \mathrm{a}$ and Figure $6 \mathrm{~b}, \mathrm{f}, \mathrm{j}, \mathrm{n}$ ). On the other hand, higher toxicity was observed in the cells incubated with L-NH 4 -EDTA $(20: 1)$ or L-MnSO 4 (20:1), compared to L-NH4-EDTA (2:1) and L-MnSO 4 (2:1), respectively (Figure $7 \mathrm{~b}, \mathrm{c}, \mathrm{e}, \mathrm{f}$ ). Furthermore, the resazurin assay showed a significant difference in the viability of cells treated with L-NH4-EDTA 2:1 (85\%) (Figure 7 e) and L-MnSO $2: 1$ (77\%) (Figure $7 \mathrm{f}$ ) compared with $\mathrm{L}-\left(\mathrm{NH}_{4}\right)_{2} \mathrm{SO}_{4}$ 2:1 (61\%) (Figure $7 \mathrm{~d}$ ), where the latter showed the lowest cell viability at 2:1 ratio. These results are in agreement with the flow cytometry and CLSM results, where higher drug release in combination with $\mathrm{HT}$ led to higher cellular uptake and toxicity.

\section{Remote loading methods and lipid:DOX ratio influence DOX-loaded LTSLs in vivo release}

In order to evaluate the in vivo release behaviour of DOX-loaded LTSLs using different remote loading methods and lipid:DOX weight ratios, L- $\left(\mathrm{NH}_{4}\right)_{2} \mathrm{SO}_{4}(20: 1)$, L- $\left(\mathrm{NH}_{4}\right)_{2} \mathrm{SO}_{4}(2: 1), \mathrm{L}-\mathrm{NH}_{4}-\mathrm{EDTA}(20: 1)$ and L-NH 4 -EDTA (2:1) were injected intravenously at $8 \mathrm{mg} \mathrm{DOX} / \mathrm{kg}$ in bifocal tumour (CT26)-bearing mice.

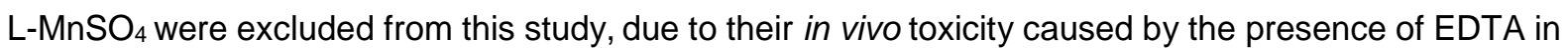
the sample, where attempts of buffer exchange resulted in unstable liposomes (data not shown). The effect of localised HT on DOX-loaded LTSLs' delivery and release was assessed by immersing one 
tumour-bearing leg in a $42{ }^{\circ} \mathrm{C}$ water bath for 1 hour $(+\mathrm{HT})$ immediately after liposomes injection. The other tumour-bearing leg was used as a non-heated control (-HT).

\section{Figure 8}

Figure 8a shows whole-body fluorescence images of CT26-tumour bearing mice intravenously injected with DOX-loaded LTSLs combined with localised mild HT in the tumour-bearing leg. Immediately after 1 hour of $\mathrm{HT}$, the highest fluorescence signals were observed in tumours treated with $\mathrm{L}-\left(\mathrm{NH}_{4}\right)_{2} \mathrm{SO}_{4}$ (20:1) and L-NH 4 -EDTA (20:1), followed by $\mathrm{L}-\left(\mathrm{NH}_{4}\right)_{2} \mathrm{SO}_{4}$ (2:1), whilst no DOX fluorescence signals were observed in mice injected with L-NH$H_{4}$-EDTA (2:1). 24 hours post-HT, no significant changes in DOX signals were detected, except for a slight increase in the fluorescence signal of $\mathrm{L}-\left(\mathrm{NH}_{4}\right)_{2} \mathrm{SO}_{4}$ (2:1) group, which could be attributed to the enhanced liposomes' tumour accumulation following HT, as previously reported [25]. Figures $8 \mathrm{~b} \& \mathrm{c}$ show the quantification of the tumour region of interest (ROI)'s fluorescence at 1 and 24 hours after the treatment. One-hour post-HT, about $\sim 2$-fold fluorescence increase was detected in mice injected with $\mathrm{L}-\left(\mathrm{NH}_{4}\right)_{2} \mathrm{SO}_{4}(20: 1)$, $\mathrm{L}-\left(\mathrm{NH}_{4}\right)_{2} \mathrm{SO}_{4}(2: 1)$ and L-NH $4-E D T A$ (20:1), compared with non-heated tumours. Surprisingly, L-NH4-EDTA (2:1) showed a very low fluorescence signal in the $+\mathrm{HT}$ tumour side with comparable values to the $-\mathrm{HT}$ side, suggesting an incomplete or slower drug release of this formulation. After 24 hours (Figure $8 \mathrm{c}$ ), DOX fluorescence signals were maintained in heated tumours and the accumulation of DOX was slightly increased in the non-heated tumours compared to the 1-hour time point.

After 24 hours, ex vivo analysis of the organs was carried out. Negligible DOX uptake was detected in lungs, heart and spleen (Figure $8 \mathrm{~d} \& \mathrm{e}$ ). As expected, higher DOX levels were observed in the liver and kidneys compared with the control group since the LTSLs exhibit lower stability in serum than traditional formulations [25]. The effect of localised mild HT versus the enhanced permeation and retention effect can be observed in the graph, where a significant increase in DOX accumulation ( 2.5fold change increase) was detected in the heated tumours compared with the non-heated ones. This increase in the fluorescence in heated tumours reflects DOX release from the LTSLs at $42{ }^{\circ} \mathrm{C}$ (Figure 4 a-d). More importantly, groups treated with HT, L- $\left(\mathrm{NH}_{4}\right)_{2} \mathrm{SO}_{4}(20: 1)$, L-NH $4-E D T A(20: 1)$, and L$\left(\mathrm{NH}_{4}\right)_{2} \mathrm{SO}_{4}(2: 1)$ had more efficient tumour DOX release compared with the L-NH 4 -EDTA (2:1) group. These results agree with the in vitro uptake experiments, where DOX release/uptake of L-NH 4 -EDTA (2:1) was significantly diminished in the presence of $50 \%$ serum compared with the other LTSLs formulations.

\section{Remote loading methods and lipid:DOX ratio influence DOX-loaded LTSLs anticancer activity in vivo}

We next examined the therapeutic efficacy of the various DOX-loaded LTSLs in combination with mild $\mathrm{HT}$ in vivo (Figure 9). CT26 tumour-bearing mice were injected intravenously with $\mathrm{L}-\left(\mathrm{NH}_{4}\right)_{2} \mathrm{SO}_{4}(20: 1)$, 
L- $\left(\mathrm{NH}_{4}\right)_{2} \mathrm{SO}_{4}(2: 1)$, L-NH 4 -EDTA (20:1) or L-NH 4 -EDTA (2:1) at $5 \mathrm{mg}$ Dox/kg. Following injection, the tumour-bearing leg was immediately immersed in a $42{ }^{\circ} \mathrm{C}$ water bath for 1 hour. Figure 9 a shows a significant delay in the tumour growth in mice treated with L-( $\left.\mathrm{NH}_{4}\right)_{2} \mathrm{SO}_{4}(20: 1)$, L-NH $4-E D T A(20: 1)$ and $\mathrm{L}-\left(\mathrm{NH}_{4}\right)_{2} \mathrm{SO}_{4}$ (2:1), whilst L-NH 4 -EDTA (2:1) did not show significant differences compared with the control up to 22 days after tumour inoculation, due to inefficient DOX release (Figure 8 a). Nevertheless, all survival curves' results were significantly different from the control which had a median survival of 21 days $\left({ }^{* *} \mathrm{p}<0.01\right)$ (Figure $\left.9 \mathrm{~b}\right)$. The group that received $\mathrm{L}-\left(\mathrm{NH}_{4}\right)_{2} \mathrm{SO}_{4}(20: 1)$ showed the longest median survival of 60 days, followed by L-NH4-EDTA $(20: 1)$ and $\mathrm{L}-\left(\mathrm{NH}_{4}\right)_{2} \mathrm{SO}_{4}(2: 1)$ with a median survival of 30 and 39 , respectively. As anticipated, L-NH 4 -EDTA (2:1) group showed the shortest median survival of 26. No signs of toxicity were observed from all treated groups and no significant change in the body weight of the mice was observed across the study (Figure SI 2).

Figure 9

\section{Discussion}

TSLs have been a promising delivery system to alter a drug's pharmacokinetics and increase its bioavailability at targeted tissues, while reducing its systemic toxicity. Over the years, extensive work has advanced TSLs, such as by pegylation to prolong blood circulation [15], or varying lipid composition to tune drug release [10], [16], [17], or including lysolipids to accelerate drug release [9]. ThermoDox ${ }^{\circledR}$ is the most advanced LTSLs formulation with ultrafast release kinetics when mild HT is applied (41$42^{\circ} \mathrm{C}$ ). The majority of published studies reported DOX-loaded LTSLs using the well-established ammonium sulphate gradient at high lipid:drug ratios $(20: 1$ or $10: 1)$. Currently, there are no reports assessing the effect of different remote loading methods or lipid:drug ratios on the anticancer activity of LTSLs. Interestingly, our findings demonstrate for the first time the links between the nature of DOX crystals and the thermosensitivity and therapeutic efficacy of DOX-LTSLs in vitro and in vivo.

Optimal loading of weak bases, such as DOX, into LTSLs has been achieved via remote loading methods, which rely on the generation of a transmembrane $\mathrm{pH}$-gradient to drive efficient DOX loading inside the liposome [31]. In general, as free DOX diffuses into the liposomes, it becomes protonated in the acidic intraliposomal environment, which inhibits its membrane re-permeation [32]. Besides the ammonium sulphate, other remote loading methods of anthracyclines have been reported. For instance, Song et al., demonstrated efficient DOX loading into NTSLs using ammonium EDTA [24]. pH-gradient remote loading methods have been developed to stably and efficiently encapsulate anthracyclines (DOX, idarubicin or daunorubicin) into conventional liposomal formulations, such as Doxil ${ }^{\circledR}$ and Myocet ${ }^{\circledR}$ [24], [33]. However, lower DOX loading capacity, with an optimum 20:1 lipid:DOX weight ratio, has often been reported when loading DOX into the LTSL formulation (ThermoDox ${ }^{\circledR}$ ) [9], [32], [34], [35]. In the present work, we have investigated the effect of different $\mathrm{pH}$-gradient remote loading methods, namely $\left(\mathrm{NH}_{4}\right)_{2} \mathrm{SO}_{4}$ and $\mathrm{NH}_{4}$-EDTA on DOX loading into LTSLs. Our results showed that, for the first time, $\mathrm{NH}_{4}$ EDTA remote loading into LTSLs was similar to the commonly used $\left(\mathrm{NH}_{4}\right)_{2} \mathrm{SO}_{4}$ method. The mechanism 
of efficient DOX loading via $\left(\mathrm{NH}_{4}\right)_{2} \mathrm{SO}_{4}$ has been based on the low solubility of needle-like, DOXsulphate crystals inside liposomes [36], [37]. On the other hand, the mechanism of DOX loading with $\mathrm{NH}_{4}$-EDTA is not well understood yet. We believe that the positive charge of the protonated DOX in the intraliposomal acidic compartment interacts electrostatically with the negative charges of the carboxyl group of the EDTA, leading to circular DOX crystals formation (Figure 2) similar to DOX-citrate bundles [38]. Importantly, our results showed high stability of all DOX-loaded LTSLs using the $\mathrm{NH}_{4}$-EDTA over one month, with minimum changes in their colloidal properties (data not shown). In contrast, L$\left(\mathrm{NH}_{4}\right)_{2} \mathrm{SO}_{4}$ showed an increase in size and PDI at low lipid:drug ratios $(<5: 1)$, which justifies 20:1 lipid:DOX weight ratio as the optimal ratio for a stable DOX-LTSLs formulation. The superior stability of L-NH 4 -EDTA has been attributed to EDTA chelating properties, which prevent phospholipid oxidation [24]. It is thought that DOX planar aromatic rings stack longitudinally in a hexagonal arrangement at low $\mathrm{pH}$ [39], [40], which leads to linear rod-like fibres in the presence of the sulphate ions and gives a "coffee bean" appearance. Cholesterol-containing liposomes are known to withstand the deformity at high DOX encapsulation efficiency, maintaining high DOX loading. On the other hand, cholesterol-free LTSLs do not withstand these structural changes, which leads to their deformation and rupture at lipid:DOX weight ratios lower than 10:1, resulting in lower long-term stability. In contrast, L-NH4-EDTA (2:1) demonstrated efficient DOX loading and longer stability, which could be attributed to the flexible circular crystals (Figure 2, Figure 3).

DOX loading into liposomes via metal complexation, such as manganese or copper, has been an alternative to the $\mathrm{pH}$-gradient remote loading methods [20], [26], [41]. Entrapped divalent ions inside the liposome's aqueous core form stable complexes with DOX, preventing their re-permeation. The purple colour of $\mathrm{L}-\mathrm{MnSO}_{4}$ indicates the successful encapsulation of the complexes into LTSLs, as reported by others [42], [20], [21]. However, our lower EE\% contrasts with previous reports using conventional [21] and LTSL formulations [26]. Cheung et al., reported high DOX EE\% ( 100\%) using conventional sphingomyelin/cholesterol [20]. Chiu et al., efficiently loaded DOX into LTSLs via manganese complexation to improve DOX loading into LTSLs and overcome their inherent leakiness in the blood [26]. Other authors exploited DOX-Mn²+ complexes as an MRI contrast agent using LTSLS [43] and TSLs [44], but no EE values were reported. Our L-MnSO 4 revealed a slower release profile compared to other DOX-loaded LTSLs formulations. Furthermore, the microscopic examination of L$\mathrm{MnSO}_{4}$ revealed faceted-shaped liposomes, with an empty-looking core [26], which could explain their lower stability at $37^{\circ} \mathrm{C}$ in HBS buffer. Moreover, $\mathrm{L}^{-\mathrm{MnSO}_{4}}$ formulation was excluded from the in vivo studies due to the toxicity associated with the high concentration of EDTA [45], [46], indicating their unsuitability for clinical applications.

Developing ultrafast releasing formulations like ThermoDox ${ }^{\circledR}$ is crucial to enabling intravascular DOX release in heated tumours [9]. This improves DOX penetration and accumulation not only in tumour cells but also stromal and endothelial cells and pericytes, as reported by others [10]. Most of the previous studies have utilized in vitro drug release profiles in the presence of serum proteins to evaluate the thermosensitive nature of TSLS and the rate of drug release. However, these studies do not 
completely reflect the actual release scenario in in vivo conditions. In the present work, we have thoroughly investigated the release of DOX-loaded LTSLs, prepared using different loading buffers and DOX content, in in vitro and in vivo models with various degrees of complexity. It was evident that by increasing the complexity of the model used, the impact of the remote loading methods and the lipid:DOX ratio on DOX-LTSLs' therapeutic efficacy was more pronounced. To our knowledge, this is the first report correlating in vitro testing with in vivo release and therapeutic efficacy, and not simply using serum-containing media as often described [9], [20], [24], [47]. Our results showed that changing lipid:DOX ratios, while using the same loading method, did not guarantee similar in vitro and in vivo therapeutic efficacy of DOX-LTSLs, as evidenced by the slower release of L-NH4-EDTA (2:1) compared to L-NH 4 -EDTA (20:1) (Figure $5 \&$ 8). Similarly, $\mathrm{L}-\left(\mathrm{NH}_{4}\right)_{2} \mathrm{SO}_{4}(2: 1)$ showed lower mice survival rates compared to the $\mathrm{L}-\left(\mathrm{NH}_{4}\right)_{2} \mathrm{SO}_{4}(20: 1)$ group (Figure 8), which could support the selection of 20:1 lipid:DOX ratio as the optimal ratio for the clinically tested ThermoDox ${ }^{\circledR}$. Banno et al. reported that lysolipid desorption from LTSLs influences their release profile in vivo [48]. A rapid loss of lysolipid molecules (from $9.6 \%$ to $3.6 \%$ ) from LTSLs was observed within the first 10 minutes of intravenous administration, where lysolipid loss continued over time. This finding emphasises the importance of combining fast dissolution and release of DOX crystals loaded into LTSLs whilst the lysolipid is still incorporated in the lipid bilayer, to ensure intravascular drug release in combination with mild HT. The present study has confirmed that the thermosensitivity of TSL formulations is not solely determined by the lipid bilayer composition, since DOX release kinetics from the LTSLs were dictated by the nature of the crystal/precipitate (i.e., bundle, rings) inside the liposomes and their conformational rearrangement during HT (Figure 2). Therefore, factors influencing DOX crystallisation should be taken into account whilst designing ultrafast thermoresponsive nanocarriers.

Different remote loading methods and lipid:DOX ratios were interchangeably used to load Doxil ${ }^{\circledR}$-like formulations. However, the high stability of these formulations and their slow release profile did not significantly impact the efficacy of these conventional liposomes in vitro and in vivo, unlike DOX-LTSLs. Previously, we showed minimal effects of DOX content on the pharmacokinetics, biodistribution and tumour accumulation of a Doxil ${ }^{\circledR}$-like formulation [25]. The high stability of Doxi ${ }^{\circledR}$ formulation could withstand the enlargement of DOX crystals without affecting its pharmacokinetics. The latter differs from cholesterol-free LTSLs, where LTSLs with high DOX content could exhibit different pharmacokinetics and behaviour in vivo. The high content of DOX inside LTSLs could affect the rigidity/shape of the liposomes and their interaction with blood components (serum proteins and immune cells) following systemic administration, resulting, probably, in faster blood clearance. The shorter blood circulation combined with lysolipid desorption and slower DOX release, could explain the lower therapeutic efficacy of both formulations loaded at a low lipid:DOX (2:1) ratio. However, other factors, such as altered pharmacokinetics profiles, serum stability, uptake by the immune cells, and tumour extravasation and tissue distribution, could contribute to the therapeutic efficacy of DOX-LTSLs in vivo, and should be further investigated. Nevertheless, despite the lower therapeutic efficacy of L-( $\left.\mathrm{NH}_{4}\right)_{2} \mathrm{SO}_{4}(2: 1)$ and L$\mathrm{NH}_{4}$-EDTA (2:1) co-administered with mild $\mathrm{HT}$, we believe that their anticancer activity could be 
modulated based on the HT regimen (single or multiple mild $\mathrm{HT}$ ) used to induce in vivo intravascular or/and interstitial release.

\section{Conclusions}

This study highlights an alternative approach to manipulate the thermosensitivity of TSLs via modifying DOX crystals, rather than the lipid bilayer composition. In this work we have thoroughly investigated different remote loading methods $\left(\left(\mathrm{NH}_{4}\right)_{2} \mathrm{SO}_{4}, \mathrm{NH}_{4}-\mathrm{EDTA}, \mathrm{MnSO}_{4}\right)$ to load DOX into LTSLs. More importantly, we demonstrated the physicochemical properties of DOX crystals in the core of the LTSLS vary depending on the loading method and lipid:DOX ratio used, and how these physicochemical properties could influence the in vitro and in vivo LTSLs release profile, uptake and toxicity in cancer cells. Overall, our findings could be relevant to a range of triggerable liposomal formulations where their therapeutic efficacy has been limited by an undesirable release profile.

\section{Acknowledgments}

This work was supported by the Prostate Cancer UK (Grant CDF12-002), The Engineering and Physical Sciences Research Council (EPSRC) (EP/M008657/1), The Royal Society (RG2014 R1), and Queen's University Belfast. The authors would like to thank Mr. Robert Pike for proofreading the manuscript.

\section{References}

[1] G. Tiwari et al., "Drug delivery systems: An updated review," Int. J. Pharm. Investig., vol. 2, no. 1, p. 2, Jan. 2012.

[2] Y. Barenholz, "Doxil-the first FDA-approved nano-drug: lessons learned," J. Control Release, vol. 160, no. 2, pp. 117-134, 2012.

[3] L. A. Smith et al., "Cardiotoxicity of anthracycline agents for the treatment of cancer: Systematic review and meta-analysis of randomised controlled trials," BMC Cancer, vol. 10, no. 1, p. 337, Dec. 2010.

[4] M. Gyöngyösi et al., "Liposomal doxorubicin attenuates cardiotoxicity via induction of interferonrelated DNA damage resistance," Cardiovasc. Res., vol. 116, no. 5, pp. 970-982, Jul. 2019.

[5] K. M. Laginha, S. Verwoert, G. J. R. R. Charrois, and T. M. Allen, "Determination of Doxorubicin Levels in Whole Tumor and Tumor Nuclei in Murine Breast Cancer Tumors," Clin. Cancer Res., vol. 11, no. 19, pp. 6944 LP - 6949, Oct. 2005.

[6] E. Oude Blenke, E. Mastrobattista, and R. M. Schiffelers, "Strategies for triggered drug release from tumor targeted liposomes," Expert Opin. Drug Deliv., vol. 10, no. 10, pp. 1399-1410, Oct. 2013.

[7] M. B. Yatvin, J. N. Weinstein, W. H. Dennis, and R. Blumenthal, "Design of liposomes for 
enhanced local release of drugs by hyperthermia," Science (80-. )., vol. 202, no. 4374, pp. 1290 LP - 1293, Dec. 1978.

[8] G. R. Anyarambhatla and D. Needham, "Enhancement of the phase transition permeability of DPPC liposomes by incorporation of MPPC: A new temperature-sensitive liposome for use with mild hyperthermia," J. Liposome Res., vol. 9, no. 4, pp. 491-506, 1999.

[9] D. Needham, G. Anyarambhatla, G. Kong, and M. W. Dewhirst, "A New Temperature-sensitive Liposome for Use with Mild Hyperthermia: Characterization and Testing in a Human Tumor Xenograft Model," Cancer Res., vol. 60, no. 5, pp. 1197 LP - 1201, Mar. 2000.

[10] D. Needham, J. Y. Park, A. M. Wright, and J. Tong, "Materials characterization of the low temperature sensitive liposome (LTSL): Effects of the lipid composition (lysolipid and DSPEPEG2000) on the thermal transition and release of doxorubicin," Faraday Discuss., vol. 161, pp. 515-534, 2013.

[11] H. I. Chang and M. K. Yeh, "Clinical development of liposome-based drugs: Formulation, characterization, and therapeutic efficacy," Int. J. Nanomedicine, vol. 7, pp. 49-60, 2012.

[12] U.S. National Library of Medicine, "Clinicaltrials.gov." [Online]. Available: https://clinicaltrials.gov/ct2/home.

[13] P. C. Lyon et al., "Clinical trial protocol for TARDOX: a phase I study to investigate the feasibility of targeted release of lyso-thermosensitive liposomal doxorubicin (ThermoDox®) using focused ultrasound in patients with liver tumours," J. Ther. Ultrasound, vol. 5, no. 1, p. 28, Dec. 2017.

[14] "A Phase I Study of Lyso-thermosensitive Liposomal Doxorubicin and MR-HIFU for Pediatric Refractory Solid Tumors," NCT02536183. [Online]. Available: https://clinicaltrials.gov/ct2/show/NCT02536183?cond=thermodox\&draw=2\&rank=7.

[15] M. H. Gaber, K. Hong, S. K. Huang, and D. Papahadjopoulos, "Thermosensitive Sterically Stabilized Liposomes: Formulation and in Vitro Studies on Mechanism of Doxorubicin Release by Bovine Serum and Human Plasma," Pharm. Res., vol. 12, no. 10, pp. 1407-1416, 1995.

[16] L. Li et al., "Triggered content release from optimized stealth thermosensitive liposomes using mild hyperthermia," J. Control. Release, vol. 143, no. 2, pp. 274-279, Apr. 2010.

[17] W. J. M. Lokerse, E. C. M. Kneepkens, T. L. M. ten Hagen, A. M. M. Eggermont, H. Grüll, and G. A. Koning, "In depth study on thermosensitive liposomes: Optimizing formulations for tumor specific therapy and in vitro to in vivo relations," Biomaterials, vol. 82, pp. 138-150, Mar. 2016.

[18] Z. S. Al-Ahmady, O. Chaloin, and K. Kostarelos, "Monoclonal antibody-targeted, temperaturesensitive liposomes: In vivo tumor chemotherapeutics in combination with mild hyperthermia," J. Control. Release, vol. 196, pp. 332-343, Dec. 2014.

[19] Z. S. Al-Ahmady, C. L. Scudamore, and K. Kostarelos, "Triggered doxorubicin release in solid tumors from thermosensitive liposome-peptide hybrids: Critical parameters and therapeutic efficacy," Int. J. Cancer, vol. 137, no. 3, pp. 731-743, Aug. 2015.

[20] B. C. L. Cheung, T. H. T. Sun, J. M. Leenhouts, and P. R. Cullis, "Loading of doxorubicin into liposomes by forming Mn2+-drug complexes," Biochim. Biophys. Acta - Biomembr., vol. 1414, no. 1-2, pp. 205-216, 1998.

[21] S. A. Abraham et al., "Formation of transition metal-doxorubicin complexes inside liposomes," 
Biochim. Biophys. Acta - Biomembr., vol. 1565, no. 1, pp. 41-54, 2002.

[22] J. Gubernator et al., "The encapsulation of idarubicin within liposomes using the novel EDTA ion gradient method ensures improved drug retention in vitro and in vivo," J. Control. Release, vol. 146, no. 1, pp. 68-75, 2010.

[23] N. Forbes, A. Pallaoro, N. O. Reich, and J. A. Zasadzinski, "Rapid, Reversible Release from Thermosensitive Liposomes Triggered by Near-Infra-Red Light," Part. Part. Syst. Charact., vol. 31, no. 11, pp. 1158-1167, Nov. 2014.

[24] Y. Y. Song et al., "The application of EDTA in drug delivery systems: doxorubicin liposomes loaded via NH4EDTA gradient," Int. J. Nanomedicine, vol. 9, pp. 3611-3621, 2014.

[25] W. T. Al-Jamal, Z. S. Al-Ahmady, and K. Kostarelos, "Pharmacokinetics \&amp; tissue distribution of temperature-sensitive liposomal doxorubicin in tumor-bearing mice triggered with mild hyperthermia," Biomaterials, vol. 33, no. 18, pp. 4608-4617, Jun. 2012.

[26] G. N. C. Chiu et al., "Encapsulation of doxorubicin into thermosensitive liposomes via complexation with the transition metal manganese," J. Control. Release, vol. 104, no. 2, pp. 271-288, 2005.

[27] S. Nappini, S. Fogli, B. Castroflorio, M. Bonini, F. Baldelli Bombelli, and P. Baglioni, "Magnetic field responsive drug release from magnetoliposomes in biological fluids," J. Mater. Chem. B, vol. 4, no. 4, pp. 716-725, 2016.

[28] Z. S. Al-Ahmady, M. Hadjidemetriou, J. Gubbins, and K. Kostarelos, "Formation of protein corona in vivo affects drug release from temperature-sensitive liposomes," J. Control. Release, vol. 276, pp. 157-167, Apr. 2018.

[29] F. Lin, L. Hoogendijk, L. Buil, J. H. Beijnen, and O. Van Tellingen, "Sildenafil is not a useful modulator of $A B C B 1$ and $A B C G 2$ mediated drug resistance in vivo," Eur. J. Cancer, vol. 49, no. 8, pp. 2059-2064, May 2013.

[30] "The human protein atlas." [Online]. Available: https://www.proteinatlas.org/ENSG00000005471-ABCB4/cell\#gene_information.

[31] S. Sur, A. C. Fries, K. W. Kinzler, S. Zhou, and B. Vogelstein, "Remote loading of preencapsulated drugs into stealth liposomes," Proc. Natl. Acad. Sci., vol. 111, no. 6, pp. $2283-$ 2288, 2014.

[32] A. Fritze, F. Hens, A. Kimpfler, R. Schubert, and R. Peschka-Süss, "Remote loading of doxorubicin into liposomes driven by a transmembrane phosphate gradient," Biochim. Biophys. Acta - Biomembr., vol. 1758, no. 10, pp. 1633-1640, 2006.

[33] M. Chountoulesi, N. Naziris, N. Pippa, and C. Demetzos, "The significance of drug-to-lipid ratio to the development of optimized liposomal formulation," J. Liposome Res., vol. 28, no. 3, pp. 249-258, Jul. 2018.

[34] C. D. Landon, "Nanoscale Drug Delivery and Hyperthermia: The Materials Design and Preclinical and Clinical Testing of Low Temperature-Sensitive Liposomes Used in Combination with Mild Hyperthermia in the Treatment of Local Cancer," Open Nanomed. J., vol. 3, no. 1, pp. 24-37, May 2011.

[35] A. M. Ponce, A. Wright, M. W. Dewhirst, and D. Needham, "Targeted bioavailability of drugs by 
triggered release from liposomes," Future Lipidol., vol. 1, no. 1, pp. 25-34, Feb. 2006.

[36] G. Haran, R. Cohen, L. K. Bar, and Y. Barenholz, "Transmembrane ammonium sulfate gradients in liposomes produce efficient and stable entrapment of amphipathic weak bases," Biochim. Biophys. Acta - Biomembr., vol. 1151, no. 2, pp. 201-215, Sep. 1993.

[37] A. C. Chakrabarti, I. Clark-Lewis, P. R. Harrigan, and P. R. Cullis, "Uptake of basic amino acids and peptides into liposomes in response to transmembrane $\mathrm{pH}$ gradients," Biophys. J., vol. 61, no. 1, pp. 228-234, 1992.

[38] X. Li et al., "Doxorubicin physical state in solution and inside liposomes loaded via a pH gradient," Biochim. Biophys. Acta - Biomembr., vol. 1415, no. 1, pp. 23-40, 1998.

[39] J. B. Chaires, N. Dattagupta, and D. M. Crothers, "Self-association of daunomycin.," Biochemistry, vol. 21, no. 17, pp. 3927-3932, Aug. 1982.

[40] M. Menozzi, L. Valentini, E. Vannini, and F. Arcamone, "Self-Association of Doxorubicin and Related Compounds in Aqueous Solution," J. Pharm. Sci., vol. 73, no. 6, pp. 766-770, Jun. 1984.

[41] A. Kheirolomoom et al., "Copper-doxorubicin as a nanoparticle cargo retains efficacy with minimal toxicity," Mol. Pharm., vol. 7, no. 6, pp. 1948-1958, 2010.

[42] A. Jabłońska-Trypuć, G. Świderski, R. Krętowski, and W. Lewandowski, "Newly Synthesized Doxorubicin Complexes with Selected Metals-Synthesis, Structure and Anti-Breast Cancer Activity," Molecules, vol. 22, no. 7, p. 1106, Jul. 2017.

[43] A. M. Ponce et al., "Magnetic resonance imaging of temperature- sensitive liposome release: Drug dose painting and antitumor effects," J. Natl. Cancer Inst., vol. 99, no. 1, pp. 53-63, 2007.

[44] S. Y. Yeo, M. De Smet, S. Langereis, L. Vander Elst, R. N. Muller, and H. Grüll, "Temperaturesensitive paramagnetic liposomes for image-guided drug delivery: Mn2 + versus [Gd(HPDO3A)(H2O)]," Biochim. Biophys. Acta - Biomembr., vol. 1838, no. 11, pp. 2807-2816, 2014.

[45] "Final Report on the Safety Assessment of EDTA, Calcium Disodium EDTA, Diammonium EDTA, Dipotassium EDTA, Disodium EDTA, TEA-EDTA, Tetrasodium EDTA, Tripotassium EDTA, Trisodium EDTA, HEDTA, and Trisodium HEDTA," Int. J. Toxicol., vol. 21, pp. 95-142, 2002.

[46] Y. Kanemaru, M. J. Rossowska, C. H. Narayanan, and T. Nakamoto, "Effect of caffeine and zinc on DNA and protein synthesis of neonatal rat cardiac muscle cell in culture," Res. Exp. Med., vol. 192, no. 1, pp. 115-122, 1992.

[47] J. Gubernator et al., "Efficient human breast cancer xenograft regression after a single treatment with a novel liposomal formulation of epirubicin prepared using the EDTA ion gradient method," PLoS One, vol. 9, no. 3, p. e91487, 2014.

[48] B. Banno et al., "The functional roles of poly(ethylene glycol)-lipid and lysolipid in the drug retention and release from lysolipid-containing thermosensitive liposomes in vitro and in vivo," J. Pharm. Sci., vol. 99, no. 5, pp. 2295-2308, May 2010. 